\section{Rede intersetorial do Programa Saúde na Escola: sujeitos, percepções e práticas}

\author{
Inter-sector network in Brazil's School Health \\ Program: subjects, perceptions, and practices
}

\section{Red intersectorial del Programa Salud en la Escuela: sujetos, percepciones y prácticas}

\author{
Antônio Paulo Gomes Chiari 1,2 \\ Raquel Conceição Ferreira 1 \\ Marco Akerman 3 \\ João Henrique Lara do Amaral 1 \\ Kecyanne Malheiros Machado 1 \\ Maria Inês Barreiros Senna 1
}

doi: 10.1590/0102-311X00104217

\section{Resumo}

Este estudo avaliou a implementação e a execução do Programa Saúde na Escola (PSE) no Município de Belo Horizonte, Minas Gerais, Brasil, os mecanismos que favorecem a ação intersetorial e a percepção dos gestores municipais sobre a intersetorialidade. Foi desenvolvido um estudo de caso por meio de pesquisa documental e da aplicação de um questionário eletrônico. A pesquisa documental analisou as portarias federais e municipais sobre o PSE publicadas no Diário Oficial da União e no Diário Oficial do Município e notícias sobre o programa publicadas no Diário Oficial do Município. Um questionário semiestruturado online foi aplicado a trinta gestores municipais. Análise de conteúdo foi usada para os dados qualitativos da pesquisa documental e do questionário. Os dados quantitativos foram interpretados por análise descritiva realizada pelo programa Stata v. 13. Mecanismos integradores foram identificados nas normativas federais e municipais e no modelo singular de PSE adotado pelo município. Esses mecanismos são potencialmente promotores da articulação permanente entre a saúde e a educação. A pesquisa com os gestores demonstrou, no entanto, uma baixa utilização desses mecanismos e o predomínio da lógica setorial nessa instância do programa. Percebeu-se uma dificuldade na construção da intersetorialidade também no desenvolvimento das ações do PSE. As potencialidades da ação intersetorial identificadas nos textos oficiais e descritas nas notícias institucionais não reverberaram nas práticas dos gestores nem na atuação sobre os territórios.

Colaboração Intersetorial; Saúde Escolar; Promoção da Saúde;

Politicas Públicas

\author{
Correspondência \\ A. P. G. Chiari \\ Rua Herculano de Freitas 1436, casa 11, Belo Horizonte, MG \\ 30441-023, Brasil. \\ apgchiari@gmail.com \\ 1 Universidade Federal de Minas Gerais, Belo Horizonte, Brasil. \\ 2 Prefeitura Municipal de Belo Horizonte, Belo Horizonte, \\ Brasil. \\ 3 Faculdade de Saúde Pública, Universidade de São Paulo, São \\ Paulo, Brasil.
}




\section{Introdução}

A abordagem dos diversos determinantes dos problemas sociais demanda novos conhecimentos e práticas das organizações 1,2,3,4,5. Ações integradas construídas de forma intersetorial podem ser a chave para uma nova dinâmica no aparato governamental e para a busca de uma sociedade mais equânime, constituindo-se em modalidade de interferência em problemas no campo das práticas sociais 2,3 .

A intersetorialidade tem se mostrado como uma estratégia de gestão pública oportuna para a melhor efetivação das políticas públicas e enfrentamento da exclusão social 5 . Apesar da sua polissemia 6, intersetorialidade pode ser conceituada como "processo de articulação de saberes, potencialidades $e$ experiências de sujeitos, grupos e setores na construção de intervenções compartilhadas, estabelecendo vínculos, corresponsabilidade e cogestão para objetivos comuns" 7. Espera-se uma interdependência, com integração e interação entre sujeitos e processos organizacionais para alcançar os resultados esperados a partir de intervenções que transcendam as ações setoriais isoladas e que tenham sido desenhadas tomando o território como sua referência 2,4,5. A ação intersetorial propicia um compartilhamento de energia e de recursos visando à ampliação de respostas em torno de um projeto comum 5 , tornando-se contraponto à cultura de processos decisórios verticalizados e da disputa por poder e orçamento, tão comuns da estrutura governamental brasileira 2,5,8. Entretanto, a intersetorialidade é ainda considerada pouco clara como modelo de gestão pública, em sua aplicabilidade e como categoria de pesquisa e avaliação 6 .

A saúde, pela base científica multidisciplinar, composição multiprofissional e proximidade com outros setores das áreas sociais, é potencialmente um setor privilegiado para o desenvolvimento de ações intersetoriais. No entanto, as práticas hegemônicas de assistência em saúde mantêm caráter fragmentado, reafirmando seu isolamento ${ }^{2}$. Investigações sobre experiências intersetoriais voltadas para a saúde pública têm demonstrado a potencialidade dessas ações na promoção da saúde e no combate às iniquidades na medida em que podem viabilizar a articulação de sujeitos e processos, efetivando políticas públicas e enfrentando a exclusão social 5,6,9,10. Revisão sistemática da literatura, realizada no Canadá com o objetivo de sintetizar as evidências de impacto de ações intersetoriais nos determinantes sociais e na equidade em saúde, concluiu que os estudos não deixam claro qual a relação entre os setores envolvidos, assim como fornecem poucos detalhes sobre os processos, contextos, acertos e desafios do trabalho intersetorial ${ }^{9}$. Recente revisão de relatos de ações intersetoriais que abordavam problemas de saúde pública no Brasil observou que nenhum dos programas analisados explicita a definição de intersetorialidade na qual estão embasados e não apresentam informações sobre sua continuidade e sustentabilidade 10. Todos esses estudos concluem pela necessidade de desenvolvimento de um maior número de pesquisas avaliativas sobre a intersetorialidade nas políticas de saúde.

O trabalho intersetorial é a estratégia proposta pelo Programa Saúde na Escola (PSE), instituído no Brasil em 2007 11. Esse programa tem como base a articulação entre escola e a rede básica de saúde visando contribuir para a formação integral dos estudantes da rede pública de educação básica por meio de ações de prevenção, promoção e atenção à saúde. Prevê também a participação de outros setores e atores sociais. O PSE propõe a organização e a oferta de serviços em uma determinada área geográfica (território) e a criação de uma rede de corresponsabilidades como garantia para a sustentabilidade das ações ${ }^{12}$. Visa à qualificação das políticas públicas por meio da racionalização do uso de recursos humanos e financeiros, evitando duplicidade de ações e viabilizando a construção de projetos prioritários com foco na equidade e no enfrentamento da exclusão social. A aproximação e a atuação conjunta dos setores saúde e educação, com a participação de estudantes e familiares, possibilitam ações concretas na abordagem das vulnerabilidades e condicionantes sociais do processo saúde-doença.

Pesquisas recentes ressaltam o caráter inovador da proposta de ação intersetorial colocada pelo PSE, ao mesmo tempo em que consideram que a sua efetivação se constitui em um desafio para os gestores 13,14,15. A proposta do PSE está centrada na gestão compartilhada garantida pelos Grupos de Trabalho Intersetoriais (GTI) nos três níveis de governo 12. Nos municípios, o Grupo de Trabalho Intersetorial Municipal (GTI-M) é a instância responsável pelas ações do PSE. Os grupos de trabalho intersetoriais têm sido descritos como potenciais facilitadores de implementação de políticas públicas intersetoriais, desde que reconhecidos como instância interinstitucional, tenham representatividade, capacidade de decisão e ação e estabeleçam processos comunicativos entre os setores, os níveis de gestão e os atores envolvidos 13,16,17,18. 
Os gestores possuem papel de destaque no processo de descentralização e de incorporação e estímulo ao uso de novas tecnologias e estratégias na administração pública 2,16,19. Akerman et al. 6 propuseram que um dos pontos de partida para o estudo de empreendimentos intersetoriais deveria ser a análise dos níveis de gestão e de produção do cuidado. Nesse sentido, verifica-se a relevância em conhecer os mecanismos propostos no âmbito do PSE para favorecer a ação intersetorial nos territórios e sua utilização pelos gestores municipais. Este estudo teve como objetivos avaliar a implementação e execução do PSE no Município de Belo Horizonte, Minas Gerais, os mecanismos que favorecem a ação intersetorial e a percepção dos gestores sobre a intersetorialidade.

\section{Metodologia}

Estudo de caso da rede intersetorial do PSE no Município de Belo Horizonte, com abordagem qualitativa e quantitativa. A rede do PSE era composta pelo GTI-M, pelas coordenações regionais (CR) em cada uma das nove Administrações Regionais, pelas unidades básicas de saúde (UBS) e pelas escolas municipais.

Para avaliar o processo de implantação e execução do PSE e os mecanismos que favorecem a ação intersetorial, foi realizada uma pesquisa documental que consistiu na identificação e análise das portarias federais e municipais sobre o PSE publicadas no Diário Oficial da União (DOU) e Diário Oficial do Município (DOM) e de notícias sobre o PSE publicadas na versão digital do DOM, no período de dezembro de 2007 a outubro de 2014. Foram incluídas as portarias que tratavam do GTI-M, instância municipal de gestão intersetorial do PSE. O material foi codificado de acordo com categorias previamente definidas por Ferreira et al. 13 e confirmadas durante a leitura do material: instituição, composição, modo de funcionamento, coordenação e competências do GTI-M. Para a busca por notícias, foi usada a palavra-chave "Programa de Saúde na Escola", e foram incluídas as que abordavam o processo de implantação e desenvolvimento do PSE. As categorias de análise utilizadas foram: evolução da implantação do programa e planejamento e execução de ações intersetoriais.

A percepção dos gestores sobre a intersetorialidade do PSE foi avaliada por meio de aplicação de um questionário semiestruturado adaptado de Ferreira 20. Contém 35 questões para avaliação das dimensões da intersetorialidade, indicadas por Ferreira et al. 13: (1) gestão do PSE; (2) desenvolvimento das ações intersetoriais; (3) monitoramento e avaliação das ações; (4) sustentabilidade do PSE. O questionário foi inserido na plataforma digital Survey Monkey, enviado por e-mail e disponibilizado de maio a junho de 2015, juntamente com o Termo de Consentimento Livre Esclarecido (TCLE). Os sujeitos foram os gestores municipais do PSE, divididos em dois grupos: (1) GTI-M formado por 12 representantes da Secretaria Municipal de Educação (SMED), sete representantes da Secretaria Municipal de Saúde (SMSA) e quatro representantes da Secretaria Municipal Adjunta de Segurança Alimentar e Nutricional (SMASAN), totalizando 23 membros 21; (2) CR do PSE das nove Administrações Regionais de Belo Horizonte, formadas por um representante da área da educação e outro da área da saúde, perfazendo 18 gestores regionais.

Dados quantitativos do questionário foram interpretados por análise descritiva empregando o programa Stata v. 13 (StataCorp LP, College Station, Estados Unidos). O método de análise de conteúdo 22 foi utilizado para análise dos dados qualitativos do questionário e dos documentos selecionados, e o software NVivo 10 (QRS International Pty, Doncaster, Austrália) foi utilizado para organização, manuseio e análise desse material.

Este estudo foi aprovado pelos Comitês de Ética em Pesquisa da Universidade Federal de Minas Gerais (no 1.020.689 de 13 de abril de 2015) e da Secretaria Municipal de Saúde de Belo Horizonte (no 1.006.181 de 31 de março de 2015). 


\section{Resultados}

\section{Implementação e execução do PSE em Belo Horizonte}

\section{- GTI-M como instância de gestão intersetorial do PSE}

No estudo documental sobre o PSE, foram identificadas 19 portarias federais e duas portarias municipais, sendo que sete se referiam ao GTI-M, cinco portarias federais (Portaria MS/GM no 1.861/2008 23, Portaria MS/GM no 3.146/2009 24, Portaria Interministerial no 3.696/2010 25, Portaria Interministerial no 1.910/2011 26 e Portaria Interministerial no 1.413/2013 27) e duas portarias municipais (Portaria Conjunta SMSA/SMED no 001/2008 28 e Portaria Conjunta SMED/SMSA/SMASAN no 003/2013 21).

Em Belo Horizonte, o PSE foi instituído em 2008, e o GTI-M era constituído por 13 componentes 28. Em 2013, a composição do GTI-M foi alterada em termos do número de representantes, e houve a inclusão da Secretaria Municipal Adjunta de Segurança Alimentar e Nutricional (SMASAN) 21. As alterações nas atribuições do GTI-M de acordo com atualização das portarias federais estão apresentadas na Tabela 1. A Portaria no 1.413/2013 27 estabeleceu atribuições mais técnicas na área de planejamento. As atribuições do GTI-M do PSE de Belo Horizonte se ampliaram (Tabela 2), e ficaram estabelecidos o mecanismo de coordenação compartilhada do GTI-M, a periodicidade das reuniões e a publicação de suas deliberações no DOM. Verifica-se que as normativas federais não tratam das formas de funcionamento do GTI-M, favorecendo arranjos locais específicos.

\section{- A rede intersetorial do PSE em Belo Horizonte: sujeitos e práticas}

O estudo documental sobre a trajetória do PSE identificou 119 notícias, das quais foram analisadas 54, que estavam publicadas em 53 edições do DOM. As notícias apresentavam, na maioria das vezes, duas seções: a primeira relatava algum evento ou atividade específica do PSE, e a segunda parte apresentava informações gerais sobre o programa, englobando seus objetivos, cobertura, tipo e número de atividades realizadas. A análise cronológica das publicações revelou o processo de implantação do PSE em Belo Horizonte, que se iniciou, em 2008, por meio de um projeto piloto e atingiu, em 2010, a cobertura de $100 \%$ da rede municipal de Ensino Fundamental diurno e da rede básica de saúde (Tabela 3). Em 2013, o programa se ampliou para as Unidades Municipais de Educação Infantil.

As ações eram desenvolvidas pelas equipes da Estratégia Saúde da Família (ESF), e as avaliações de saúde dos escolares eram realizadas nas UBS. Em 2010, emergiu um novo sujeito no PSE, o monitor, sendo um para cada escola. A justificativa usada pelos gestores municipais foi que "a inserção de novos participantes amplia e fortalece o alcance das politicas de Educação e Saúde e, ao mesmo tempo, é uma oportunidade rara de formação através do trabalho para estes jovens" 29. Em 2011, ocorreu a formação das Equipes de Saúde na Escola - compostas por um enfermeiro e um auxiliar de enfermagem - que tinham como objetivos "qualificar o primeiro atendimento" e "ampliar o número de alunos atendidos" 30 . Em 2012, já atuavam vinte Equipes de Saúde na Escola, denominadas como "equipes volantes de saúde". Monitores e equipes volantes de saúde constituem as "Equipes do PSE”, e o monitor foi a categoria profissional mais citada nas notícias publicadas sobre o PSE. Os temas mais recorrentes foram as capacitações dos trabalhadores do PSE $(23 / 54=43,4 \%)$, com ênfase nas ações voltadas para o monitor $(13 / 23=56,5 \%)$ e as ações realizadas com os estudantes.

As notícias destacaram as avaliações clínicas anuais de saúde, oftalmológicas e nutricionais e as ações de saúde bucal. Atividades de promoção da saúde e prevenção também foram noticiadas em menor frequência. Publicou-se que os temas de saúde foram incluídos nos Projetos Político-pedagógicos (PPP) das escolas e que foram abordados por meio de estratégias pedagógicas diversas, tais como oficinas, rodas de conversa, projetos, feiras, seminários, gincanas, palestras e campanhas, produção de material didático com destaque para o Guia de Promoção à Saúde. Percebeu-se uma descentralização da gestão do PSE, com a descrição de 34 eventos promovidos pelas Administrações Regionais. Porém, não foram encontradas notícias referentes ao GTI-M e às coordenações regionais nem à participação de estudantes e familiares nas etapas de planejamento das ações do PSE. Observou-se que a maior parte das entrevistas publicadas foi realizada com gestores do nível central e regional das secretarias de governo envolvidas. E, nesse sentido, verificou-se que o discurso institucional considera que "o PSE 


\section{Tabela 1}

Atribuições do Grupo de Trabalho Intersetorial Municipal (GTI-M) segundo as normativas do Governo Federal. Brasil, $2008-2014$.

\begin{tabular}{|c|c|}
\hline Portarias Federais & Atribuições do GTI-M \\
\hline Portaria MS/GM no 1.861/2008 23 e Portaria MS/ & Elaborar o projeto do PSE. \\
\hline \multirow[t]{2}{*}{ GM no 3.146/2009 24} & Elaborar o termo de adesão ao PSE. \\
\hline & Enviar o projeto do PSE, em meio eletrônico, para o site do Ministério da Saúde. \\
\hline \multirow[t]{3}{*}{ Portaria Interministerial no 3.696/2010 25} & Elaborar o projeto do PSE. \\
\hline & $\begin{array}{l}\text { Articular e apoiar a implantação e implementação das ações do programa no território de } \\
\text { responsabilidade. }\end{array}$ \\
\hline & $\begin{array}{c}\text { Alimentar o cadastro secretaria e monitorar o preenchimento e a atualização dos cadastros } \\
\text { escola e unidade local integrada, todos componentes do Sistema Integrado de Monitoramento } \\
\text { do Ministério da Educação (SIMEC). }\end{array}$ \\
\hline Portaria Interministerial no 1.910/2011 26 & Executar a gestão do recurso, acompanhar e monitorar as ações do PSE no território. \\
\hline \multirow[t]{11}{*}{ Portaria Interministerial no 1.413/2013 27} & $\begin{array}{l}\text { Apoiar a implementação dos princípios e diretrizes do PSE no planejamento, monitoramento, } \\
\text { execução, avaliação e gestão dos recursos financeiros. }\end{array}$ \\
\hline & $\begin{array}{l}\text { Articular a inclusão dos temas relacionados às ações do PSE nos projetos político-pedagógicos } \\
\text { das escolas. }\end{array}$ \\
\hline & $\begin{array}{l}\text { Definir as escolas públicas a serem atendidas no âmbito do PSE, considerando-se as áreas de } \\
\text { vulnerabilidade social, os territórios de abrangência das equipes de atenção básica e os critérios } \\
\text { indicados pelo governo federal. }\end{array}$ \\
\hline & $\begin{array}{l}\text { Possibilitar a integração e planejamento conjunto entre as equipes das escolas e as equipes de } \\
\text { atenção básica. }\end{array}$ \\
\hline & Subsidiar o processo de assinatura do Termo de Compromisso pelos secretários municipais de \\
\hline & saúde e de educação. \\
\hline & $\begin{array}{l}\text { Participar do planejamento integrado de educação permanente e formação continuada e } \\
\qquad \text { viabilizar sua execução. }\end{array}$ \\
\hline & Apoiar, qualificar e garantir o preenchimento do Sistema de Monitoramento e Avaliação do PSE. \\
\hline & Propor estratégias específicas de cooperação entre Estados e Municípios para a implementação \\
\hline & e gestão do cuidado em saúde dos educandos no âmbito municipal. \\
\hline & $\begin{array}{c}\text { Garantir que os materiais do PSE, enviados pelo Ministério da Educação, sejam entregues e } \\
\text { utilizados de forma adequada pelas equipes de atenção básica e equipes das escolas. }\end{array}$ \\
\hline
\end{tabular}

PSE: Programa Saúde na Escola.

\section{Tabela 2}

Atribuições do Grupo de Trabalho Intersetorial Municipal (GTI-M) segundo as normativas do Governo Municipal de Belo Horizonte, Minas Gerais, Brasil, 2008-2014.

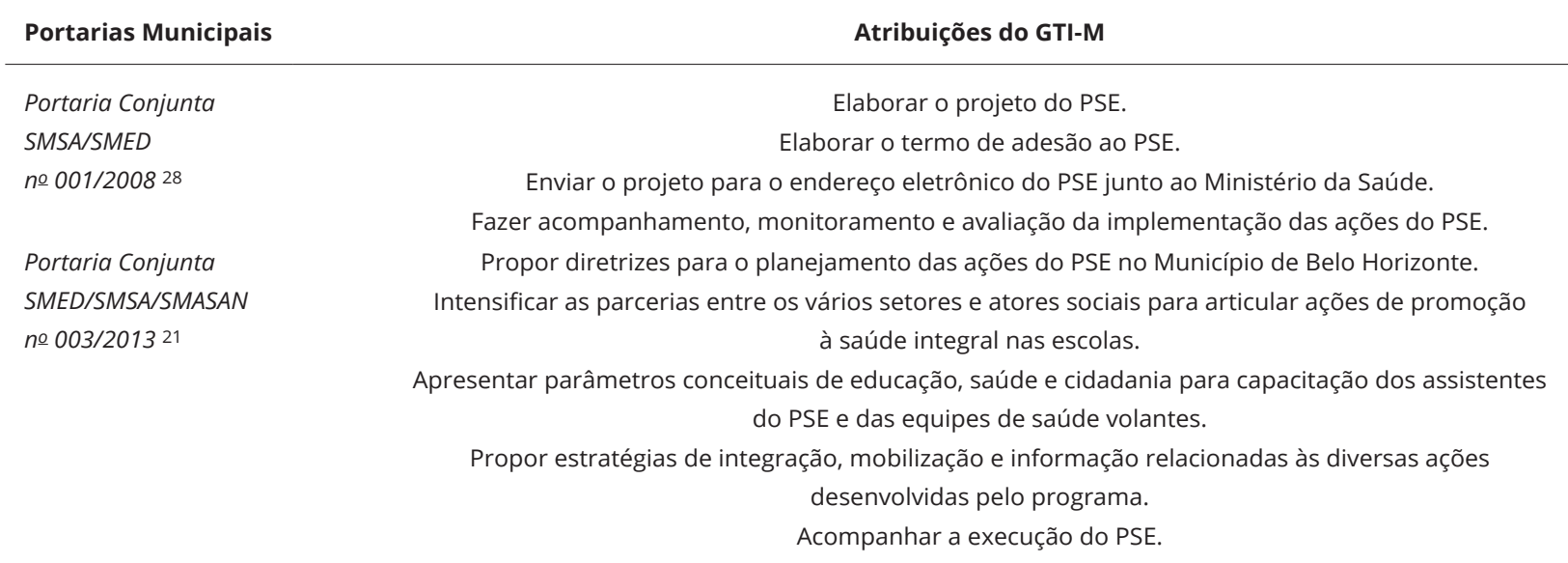

PSE: Programa Saúde na Escola. 
Tabela 3

Implantação do Programa Saúde na Escola (PSE) e número de notícias publicadas no Diário Oficial do Município (DOM). Belo Horizonte, Minas Gerais, Brasil, 2008-2014.

\begin{tabular}{|c|c|c|c|c|}
\hline Ano & $\begin{array}{c}\text { Notícias } \\
\text { publicadas } \\
\mathbf{n}\end{array}$ & $\begin{array}{c}\text { Escolas municipais } \\
\text { participantes } \\
\mathbf{n}\end{array}$ & $\begin{array}{c}\text { UBS } \\
\text { participantes } \\
\mathbf{n}\end{array}$ & $\begin{array}{c}\text { Alunos avaliados } \\
\text { (6-14 anos) } \\
n\end{array}$ \\
\hline 2008 & 1 & 9 & 9 & 3.353 \\
\hline 2009 & 1 & 36 & 61 & 6.013 \\
\hline 2010 & 9 & 169 * & $147 * *$ & 47.728 \\
\hline 2011 & 11 & 169 * & 147 ** & 82.290 \\
\hline 2012 & 7 & 169 * & 147 ** & 98.460 \\
\hline 2013 & 17 & 171 * & 147 ** & 102.395 \\
\hline 2014 & 8 & 171 * & $147 * *$ & Não é citado \\
\hline
\end{tabular}

UBS: unidades básicas de saúde.

* $100 \%$ da rede municipal de Ensino Fundamental diurno.

** $100 \%$ da rede de atenção básica.

é integrante do projeto sustentador Melhoria da Qualidade da Educação, parte do programa BH Metas e Resultados" e que "representa uma experiência bem sucedida deste governo no exercício da intersetorialidade" 31.

\section{- A rede intersetorial do PSE em Belo Horizonte: mecanismos integradores}

Os mecanismos integradores 32 identificados no arcabouço normativo do PSE, nas diretrizes para atuação e nas atribuições do GTI-M, bem como nas notícias publicadas no DOM estão sintetizados na Figura 1.

\section{Intersetorialidade no PSE: a percepção dos gestores municipais}

A taxa de participação dos gestores foi de 70\%. Dos 23 membros nomeados para o GTI-M, apenas 12 (52,2\%) mantém participação frequente e regular nas reuniões. As respondentes eram mulheres (85,7\%), com média de idade de 45 anos (DP = 12,58), com Ensino Superior (95,2\%), representantes da área da saúde $(47,6 \%)$ e com atuação no PSE há mais de quatro anos $(47,6 \%)$.

Com relação à dimensão gestão do PSE, verificou-se que poucos membros do GTI-M souberam informar a composição setorial do GTI-M, e um número menor fez referência ao mecanismo de coordenação compartilhada previsto na normativa municipal. Mais da metade do grupo não soube responder se há alternância entre os setores na coordenação do GTI-M. A Educação foi o setor mais citado como coordenador das atividades do GTI-M. Apesar de a maioria dos respondentes perceber que há equanimidade entre os setores na tomada de decisões, $15 \%$ dos respondentes tiveram opinião contrária (Tabela 4), pois alegaram que as reuniões não são deliberativas e que determinadas decisões são tomadas em instâncias superiores de gestão, para além do GTI-M. Em casos de divergências dentro do GTI-M, "a discussão [é feita] entre os gabinetes para definição dos mesmos" (GTI-M-1/Saúde). Algumas vezes "o assunto [é encaminhado] novamente para as áreas setoriais pensarem em novas estratégias" (CR-1/Educação), em busca de soluções setoriais. Esse é um ponto que gera certa tensão na percepção de gestores da área da saúde que relataram que as pautas das reuniões do GTI-M não são previamente discutidas e que essas são definidas apenas pela coordenação. Foi relatado que o grande número de componentes do GTI-M favorece uma menor participação do que a prevista na norma vigente e uma fragmentação do trabalho.

Quanto ao cumprimento das atribuições do GTI-M estabelecidas pela Portaria Interministerial no 1.413/2013 27, foram observados um significativo desconhecimento e respostas divergentes. $\mathrm{O}$ percentual de respondentes que afirmou que o GTI-M assume as atribuições de planejamento e de 
Figura 1

Mecanismos integradores identificados no Programa Saúde na Escola (PSE). Belo Horizonte, Minas Gerais, Brasil.

\begin{tabular}{|c|c|c|c|}
\hline \multicolumn{3}{|c|}{ Arcabouço normativo } & \multirow[t]{2}{*}{ Notícias publicadas no DOM } \\
\hline Federal & \multicolumn{2}{|c|}{ Municipal } & \\
\hline \multirow{3}{*}{$\begin{array}{l}\text { Capacitações conjuntas e permanentes para os } \\
\text { trabalhadores da saúde e educação }\end{array}$} & \multirow{3}{*}{$\begin{array}{l}\text { Composição do } \\
\text { GTI-M }\end{array}$} & Saúde & \multirow{3}{*}{$\begin{array}{c}\text { Equipes do PSE: } \\
\text { - Monitores (educação) } \\
\text { - Equipes volantes de saúde (saúde) }\end{array}$} \\
\hline & & Educação & \\
\hline & & Segurança alimentar & \\
\hline \multirow[t]{3}{*}{ Levantamento das necessidades do território } & \multirow{3}{*}{$\begin{array}{l}\text { Mecanismos de } \\
\text { funcionamento } \\
\text { do GTI-M }\end{array}$} & $\begin{array}{l}\text { Coordenação } \\
\text { compartilhada }\end{array}$ & \multirow{3}{*}{ Capacitações dos monitores } \\
\hline & & $\begin{array}{l}\text { Periodicidade de } \\
\text { reuniões }\end{array}$ & \\
\hline & & $\begin{array}{l}\text { Publicação das } \\
\text { deliberações }\end{array}$ & \\
\hline \multirow[t]{2}{*}{ Envolvimento dos estudantes e familiares } & \multirow{2}{*}{$\begin{array}{l}\text { Coordenações } \\
\text { regionais }\end{array}$} & Saúde & \\
\hline & & Educação & \\
\hline
\end{tabular}

DOM: Diário Oficial do Município; GTI-M: Grupo de Trabalho Intersetoruial Municipal.

monitoramento variou de $30 \%$ a $80 \%$, dependendo da atribuição. Aquelas relacionadas ao Termo de Compromisso Municipal alcançaram índice de 70\% de resposta "não sei” ao seu cumprimento pelo GTI-M. Percebeu-se uma concentração do poder decisório sobre a coordenação, já que "muitas das competências citadas são assumidas pela coordenação e não propriamente pelo grupo” (GTI-M-2/Educação). Entre os respondentes, 52,9\% não souberam precisar se a gestão do recurso financeiro do PSE é realizada de forma intersetorial.

Os modelos de gestão das áreas de saúde, educação e do PSE (Tabela 4) foram considerados como facilitadores da ação intersetorial, pois apresentam características como a participação, a descentralização, os processos de planejamento conjunto das ações e a construção de parcerias. As críticas ao setor saúde foram relativas às avaliações de saúde realizadas pelas equipes volantes nas escolas, sem a presença dos pais, fragmentando a atenção à saúde e desconsiderando o princípio da adscrição da população da ESF. Em relação ao modelo de gestão do setor educação, criticou-se que "as ações intersetoriais ainda estão muito protocolares, padronizadas, não [sendo] possível uma politica igual para todos" (GTI-M-3/Saúde). Na opinião de alguns respondentes, a educação permanece com uma gestão rígida e com pequena capacidade de flexibilização na busca por soluções para casos complexos.

A maior parte dos respondentes relatou que houve capacitação das equipes da educação e da saúde sobre o PSE, e que esse processo possibilitou a apropriação da linguagem específica dos setores (Tabela 4). Muitos, no entanto, relataram que não houve capacitações ou mesmo desconhecimento sobre elas. As respostas foram bastante divergentes em relação à participação de estudantes e seus familiares na definição de prioridades, planejamento e programação das ações do PSE. O mesmo padrão divergente entre as respostas se repete em relação ao levantamento das necessidades do território para o planejamento das ações do PSE. Para aqueles que relataram que o levantamento foi realizado, os principais responsáveis por sua execução foram as coordenações regionais do PSE $(47,1 \%)$, os profissionais das escolas $(47,1 \%)$ e os profissionais das unidades de saúde $(41,2 \%)$.

Com relação ao desenvolvimento das ações intersetoriais, os principais elementos citados para o êxito da ação intersetorial foram a integração, o planejamento conjunto, os princípios éticos e a comunicação eficaz. Entrevistado CR-2/Educação realçou a necessidade de se "conhecer as especificidades de cada setor e procurar atender as demandas apresentadas". A proximidade e integração entre as gerências 


\section{Tabela 4}

Percepção dos gestores municipais do Programa Saúde na Escola (PSE) sobre o Grupo de Trabalho Intersetorial Municipal (GTI-M) e sobre o desenvolvimento das ações intersetoriais. Belo Horizonte, Minas Gerais, Brasil, 2015.

\begin{tabular}{|c|c|c|}
\hline Variáveis selecionadas & $\mathbf{n}$ & $\%$ \\
\hline \multicolumn{3}{|l|}{ Setores representados no GTI-M } \\
\hline Educação e saúde & 13 & 61,8 \\
\hline Educação, saúde e segurança alimentar & 6 & 28,6 \\
\hline Outras composições & 2 & 9,6 \\
\hline \multicolumn{3}{|l|}{ Coordenação do GTI-M * } \\
\hline Educação & 9 & 45,0 \\
\hline Educação e saúde & 4 & 20,0 \\
\hline Saúde & 2 & 10,0 \\
\hline "Não sei" & 5 & 25,0 \\
\hline \multicolumn{3}{|l|}{ Alternância na coordenação do GTI-M } \\
\hline Sim & 3 & 14,3 \\
\hline Não & 5 & 23,8 \\
\hline "Não sei" & 13 & 61,9 \\
\hline \multicolumn{3}{|c|}{ Equanimidade na tomada de decisões no GTI-M em relação à representação dos setores participantes * } \\
\hline $\operatorname{Sim}$ & 12 & 60,0 \\
\hline Não & 3 & 15,0 \\
\hline "Não sei" & 5 & 25,0 \\
\hline \multicolumn{3}{|c|}{ Rotatividade de representantes nas reuniões e nas outras atividades do GTI-M * } \\
\hline Sim & 4 & 20,0 \\
\hline Não & 7 & 35,0 \\
\hline "Não sei" & 9 & 45,0 \\
\hline \multicolumn{3}{|c|}{ O modelo de gestão do setor saúde possibilita a ação intersetorial? ** } \\
\hline Sim & 15 & 79,0 \\
\hline Não & 1 & 5,3 \\
\hline “Não sei” & 3 & 15,7 \\
\hline \multicolumn{3}{|c|}{ O modelo de gestão do setor educação possibilita a ação intersetorial? ** } \\
\hline $\operatorname{Sim}$ & 15 & 79,0 \\
\hline Não & 2 & 10,5 \\
\hline "Não sei" & 2 & 10,5 \\
\hline \multicolumn{3}{|c|}{ O modelo de gestão do PSE possibilita a ação intersetorial? ** } \\
\hline $\operatorname{sim}$ & 17 & 89,4 \\
\hline Não & 1 & 5,3 \\
\hline “Não sei” & 1 & 5,3 \\
\hline \multicolumn{3}{|c|}{ Houve capacitação para que os profissionais da saúde e da educação compreendessem as bases estruturais do PSE? ** } \\
\hline Sim & 13 & 68,5 \\
\hline Não & 4 & 21,0 \\
\hline “Não sei” & 2 & 10,5 \\
\hline \multicolumn{3}{|c|}{ Houve capacitação para que os profissionais da saúde e da educação se apropriassem da linguagem do outro setor? ** } \\
\hline $\operatorname{sim}$ & 9 & 47,4 \\
\hline Não & 6 & 31,6 \\
\hline "Não sei” & 4 & 21,0 \\
\hline \multicolumn{3}{|c|}{ Estudantes e familiares foram envolvidos na definição de prioridades, planejamento e programação das ações do PSE? ** } \\
\hline $\operatorname{sim}$ & 7 & 36,8 \\
\hline Não & 6 & 31,6 \\
\hline “Não sei” & 6 & 31,6 \\
\hline \multicolumn{3}{|c|}{ Foi realizado levantamento das necessidades do território para as ações do PSE? *** } \\
\hline $\operatorname{Sim}$ & 10 & 58,9 \\
\hline Não & 3 & 17,6 \\
\hline “Não sei” & 4 & 23,5 \\
\hline
\end{tabular}

Fonte: questionário eletrônico (Ferreira 20) GTI-M e coordenações regionais do PSE em Belo Horizonte, 2015.

* Um participante não respondeu a essa questão $(n=20)$;

** Dois participantes não responderam a essas questões $(n=19)$;

*** Quatro participantes não responderam a essas questões $(n=17)$. 
regionais de educação e saúde foram citadas como um facilitador importante. "Pactuação não apenas no GTI, mas também nos setores de ponta [escolas e centros de saúde]” (CR-1/Saúde), foi citada como ponto fundamental para se garantir o alcance e a execução das ações. O desconhecimento sobre o programa e de seus objetivos e sobre a forma de atuação do outro setor, assim como problemas de comunicação entre os setores e entre os diferentes níveis de gestão e os profissionais da ponta do sistema foram citados como fatores dificultadores para ação intersetorial. Apontaram ainda outras dificuldades tais como incompatibilidades de agendas; excesso de atividades e número reduzido de profissionais; cumprimento de prazos e metas; planejamento centralizado; divergências e desrespeito entre os setores e dificuldade na adoção de novas posturas.

Apesar das dificuldades para o desenvolvimento do trabalho intersetorial, $76,5 \%$ dos respondentes consideraram que o PSE tem promovido cidadania e garantia de direitos humanos nos territórios de atuação. As ações mais relevantes do PSE citadas foram: oficinas sobre alimentação saudável; ações de promoção da saúde e estímulo à vida saudável; ações de saúde bucal; avaliação anual de saúde dos estudantes; ações de saúde ocular; abordagem dos alunos com dificuldade e transtorno de aprendizagem; fortalecimento do vínculo dos alunos com as unidades de saúde; melhora da interlocução entre profissionais da saúde e da educação; presença de um monitor do PSE em cada escola e ampliação do PSE para a rede de Educação Infantil. Na opinião de 64,8\% dos respondentes, esses temas da Saúde foram inseridos de forma intersetorial nos PPP das escolas.

O monitoramento e a avaliação das ações do PSE, de acordo com a maioria dos participantes, têm sido realizados por meio de instrumentos municipais elaborados pela gestão a partir das atividades realizadas pelas equipes de saúde. Apenas três respondentes citaram a utilização do Sistema Integrado de Monitoramento Execução e Controle (SIMEC), previsto na Portaria Interministerial no 1.413/201327.

As estratégias indicadas para viabilizar a sustentabilidade do PSE como política de Estado foram: ampliação e universalização das ações; ampliação de recursos para o PSE; fortalecimento da adesão de gestores e trabalhadores e das relações entre os profissionais dos diversos setores; envolvimento das famílias, da comunidade e de outras instâncias locais de participação popular.

\section{Discussão}

A implementação do PSE em Belo Horizonte avançou em termos de cobertura da rede escolar e número de estudantes atendidos. Foram identificados mecanismos integradores, potencialmente promotores da ação intersetorial e da superação da fragmentação das políticas sociais, pois geram compartilhamento de visões e formas matriciais de organização do trabalho intersetorial 32,33 . No entanto, no processo de implantação do PSE e de suas ações, esses mecanismos foram pouco utilizados, apresentando dificuldades operacionais para a adoção de uma lógica intersetorial de atuação.

As atribuições do GTI-M possibilitam a construção da articulação intersetorial. A base regional com foco em uma lógica populacional e territorial, o planejamento participativo e a descentralização são pressupostos para a implementação de ações intersetoriais. Constituem-se em paradigma orientador do modelo de gestão pública compatível com os desafios para a promoção da qualidade de vida 2,4,5,34,35. A combinação e complementaridade destes elementos - setorialidade, intersetorialidade, territorialização, democratização e participação 36 - estão presentes na proposta do PSE.

O papel ativo e central do GTI-M na condução do PSE, prescrito pela norma municipal, não se confirmou na percepção do grupo de gestores. O GTI-M apresentou-se como uma incipiente instância de gestão intersetorial: encontra-se esvaziado em relação aos membros nomeados; não se mostrou como um espaço democrático de integração capaz de promover articulação entre os atores e setores envolvidos; não apresentou poder de decisão compatível com suas atribuições; não demonstrou possuir conhecimento sobre o processo de implantação do PSE no município nem da gestão de seu recurso financeiro. Ferreira \& Silva 16 ressaltaram o papel fundamental do GTI-M no desenvolvimento do trabalho intersetorial, mas relataram que a falta de apoio institucional e de autonomia de decisão podem limitar o seu desempenho.

Neste estudo, foi desvelado um campo de tensão relacionado à coordenação e ao processo de tomada de decisões no âmbito do GTI-M. A gestão compartilhada do GTI-M, prevista na norma municipal, pode ser considerada um avanço em relação à norma federal que não trata do tema 21,27. 
No entanto, na prática, a coordenação do GTI-M é exercida pela área de Educação. E, nesse sentido, a não alternância entre os setores na coordenação do GTI-M foi considerada um ponto de conflito apenas por representantes da área da Saúde. Essa situação pode gerar diferenças percebidas e de exercício de poder, tornando-se um obstáculo para a viabilidade da intersetorialidade ${ }^{32}$. De fato, a coesão do grupo é atributo que determina seu crescimento e amadurecimento, viabilizando o cumprimento de seus objetivos 4. O GTI-M não pode prescindir de habilidades e de mecanismos formais para trabalhar os conflitos em prol da garantia do planejamento conjunto e da racionalização das intervenções 3,16,32,36,37. A prática relatada de se levar os impasses para serem resolvidos pelas áreas setoriais é a antítese da sinergia de saberes esperada na gestão intersetorial. Sinaliza uma visão setorial de um programa intersetorial 38 e a incipiência do GTI-M como espaço deliberativo e de negociação. Nesse sentido, o exercício dessas habilidades dentro do GTI-M torna-se fundamental instrumento de contraposição às disputas de poder, interesses setoriais e relações hierarquizadas, algumas das dificuldades comumente enfrentadas na construção da gestão intersetorial 32 .

Belo Horizonte construiu um modelo singular de PSE com a formação de equipes específicas para o desenvolvimento de suas ações. O termo "Equipe do PSE” foi criado para designar a equipe formada pelo monitor do PSE, enfermeiro e auxiliar de enfermagem. O monitor do PSE parece ter valorização institucional diferenciada, pois é o profissional mais citado nas notícias do DOM e para o qual está direcionado o maior investimento em capacitação/formação. Os gestores municipais o citaram de forma pontual entre as ações relevantes do programa. Já a avaliação de saúde dos estudantes, realizada nas escolas pelas equipes volantes de saúde, configurou-se como um ponto de dissenso entre os gestores. Para alguns, foi considerada um avanço do PSE municipal; para outros, constituise em um retrocesso pela atuação desvinculada do território das equipes da ESF, sem a presença dos pais dos estudantes. Neste estudo, não foi possível identificar as motivações para a construção desse desenho do PSE em Belo Horizonte. No entanto, sua rápida expansão em termos de cobertura parece estar relacionada à implantação das equipes do PSE, indicando a potencialidade do modelo. Por outro lado, a atuação das equipes da ESF e dos professores parece ser apenas marginal, de acordo com as notícias publicadas e com as respostas dos gestores. Então, cabe-nos perguntar: houve ampliação dos resultados por meio da ação intersetorial ou apenas o desenvolvimento de ações colaborativas multiprofissionais? Estudos de experiências do PSE em outras cidades brasileiras 38,39,40 demonstraram que as ações intersetoriais se sucedem de forma incipiente. Foi evidenciado que a permanência de modelos de atuação paralelos gera desgaste, desperdício, ineficiência e sobreposição no uso de recursos públicos e não altera, de forma significativa, a vida dos estudantes. O potencial inovador que as Equipes do PSE de Belo Horizonte podem trazer às práticas do programa ainda está por ser revelado.

O sucesso da ação governamental integrada depende da performance interna de cada setor, pois o paradigma intersetorial não extingue as responsabilidades setoriais específicas 6,36,41. A estrutura de gestão descentralizada das áreas temáticas no Município de Belo Horizonte é também um mecanismo integrador que possibilita a cooperação transversal entre os distintos setores 2,32,36. A gestão do PSE em Belo Horizonte, ao acompanhar essa descentralização por meio da instituição das coordenações regionais, amplia as possibilidades de articulação entre os setores envolvidos no planejamento e desenvolvimento de ações contextualizadas. Essa é uma estratégia importante em municípios de grande porte, onde as redes de educação e saúde estão distribuídas em uma grande extensão territorial com diversidade no perfil de vulnerabilidade social e de necessidades da população. No entanto, torna-se também maior o desafio da incorporação da lógica intersetorial de atuação por todas as instâncias de gestão.

As atividades assistenciais foram consideradas pelos gestores as ações mais relevantes do PSE. A análise das ações desenvolvidas pelo programa mostrou uma permanência do modelo biologicista de intervenção com um padrão tradicional de ações de saberes isolados. Não apareceu nenhuma evidência de "um novo domínio da realidade [que] traz o debate da inovação, superando o modelo de resposta pronta e única" que Sposati 36 (p. 140) chama de "nova inteligência institucional". A permanência da atuação isolada tem sido um achado relativamente frequente na análise de experiências intersetoriais: os esforços realizados podem não promover melhoria da qualidade de vida, uma vez que não ocorrem mudanças significativas na forma de desenvolvimento das ações 5,40. Por outro lado, o fortalecimento da relação e da comunicação entre escolas, unidades de saúde e estudantes, também citado pelos respondentes, 
é indício de que o PSE pode realmente trazer inovações para o campo das relações intersetoriais e da promoção de direitos de cidadania.

A inserção da saúde como tema transversal nos currículos da educação básica é uma medida assegurada pelas Diretrizes e Bases da Educação Nacional 42. Da mesma forma, é atribuição do GTI-M a articulação para inserção dos temas relacionados ao PSE nos projetos político- pedagógicos das escolas. Essas estratégias, reconhecidamente relevantes para a superação de algumas das dificuldades relatadas 38,39 , não são de construção simples. Exigem empenho coletivo dos dois setores, conhecimento mútuo e recíproco. Nesse sentido, o que tem sido realizado? O estudo com os gestores do PSE indicou que essa não é uma prática realizada por todas as escolas, assim como não há conhecimento uniforme do grupo sobre o assunto. Pela relevância estratégica do tema, esse é um campo para ações concretas dos envolvidos no programa.

As estratégias indicadas pelos gestores para consolidação do PSE como política de Estado foram, em sua maioria, etapas previstas da implementação do PSE que não foram cumpridas ou o foram de maneira fragmentada. Um processo consistente de avaliação e monitoramento também contribui para a sustentabilidade e longevidade das ações intersetoriais 41 . Alguns estudos apontaram a dificuldade em se relacionar resultados alcançados às ações intersetoriais desenvolvidas pelas políticas estudadas, indicando a fragilidade dos seus processos avaliativos 9,10 . No caso estudado, também o processo avaliativo se mostrou frágil. Os instrumentos oficiais de monitoramento do PSE são conhecidos por poucos gestores, e, ao mesmo tempo, são citados outros instrumentos elaborados pelo próprio município. Os gestores reconhecem, no entanto, que o fortalecimento do PSE pode impactar na qualidade de vida dos estudantes. Reconhecem também que esse processo de fortalecimento só será viabilizado por meio da adesão de gestores, trabalhadores, famílias e comunidades, incluindo as instâncias de participação popular já estabelecidas. Pesquisas realizadas com o PSE 13,14,38,39 e com outros programas intersetoriais 43 reforçam a necessidade de envolvimento de todos os atores no seu processo de consolidação.

O discurso institucional valoriza o PSE como uma experiência bem-sucedida de política intersetorial. Os resultados mostraram indicativos de um maior protagonismo do setor Educação na construção e no desenvolvimento do PSE no município: maior número de representantes no GTI-M; coordenação do GTI-M; destaque institucional para os monitores do PSE. Entretanto, mostraram também que as lógicas setoriais e as hierarquias de poder não foram rompidas nem na base da gestão do sistema nem nos territórios de atuação. Os mecanismos integradores identificados no arcabouço legal do PSE e nas diretrizes para a atuação do GTI-M pouco repercutiram na implementação e na execução das ações do programa. A incorporação de novas estratégias de gestão e execução das políticas públicas tem encontrado enormes dificuldades no campo das práticas.

\section{Para seguir dialogando}

Percebeu-se uma dificuldade na construção do trabalho intersetorial nos campos da gestão e do desenvolvimento das ações do PSE. Foi identificado um arcabouço normativo potencialmente promotor da intersetorialidade, tanto no âmbito federal quanto no municipal. Identificou-se também a disposição da gestão municipal para a inovação no campo da ação intersetorial por meio da implantação de um modelo singular de atuação no território. Verificou-se, no entanto, pouco compartilhamento de responsabilidades entre os setores, com pequeno alcance no desenvolvimento da promoção da saúde e na atuação sobre seus determinantes. Predominou a lógica setorial nas instâncias superiores de gestão com centralidade de poder e baixa utilização de mecanismos integradores. Os dissensos e as tensões percebidos não foram capazes de disparar a articulação dos setores/atores em prol de soluções compartilhadas para o desenvolvimento do programa. As potencialidades da ação intersetorial identificadas nos textos oficias e descritas nas notícias institucionais sobre o PSE não reverberaram nas práticas dos gestores nem na atuação sobre os territórios.

Nesse cenário, o estabelecimento de processos comunicativos entre os setores é tarefa primordial. A agenda institucional precisa ser reorganizada para que saúde e educação possam, de fato, aproximar-se. Mais do que isso, a agenda intersetorial precisa ser alçada à relevância política necessária para fazer valer o investimento realizado pelo município. Os mecanismos integradores identificados são 
potencialmente capazes de interferir positivamente na vida dos estudantes. Prioridade deve ser dada aos processos de capacitação e educação permanente que incluam a discussão atual sobre a intersetorialidade. Especial atenção deve estar voltada aos gestores locais e trabalhadores da saúde e da educação tendo em vista seu papel decisivo na realização das ações. E, nesse processo, a participação de estudantes e da comunidade é de fundamental importância para garantir a sustentabilidade das ações.

\section{Colaboradores}

A. P. G. Chiari participou da concepção e desenho do estudo, análise e interpretação dos dados e redação do manuscrito. R. C. Ferreira colaborou no desenho do estudo, análise e interpretação dos dados e revisão crítica do manuscrito. $M$. Akerman contribuiu na revisão crítica do manuscrito. J. H. L. Amaral e K. M. Machado contribuíram na análise e interpretação dos dados e revisão crítica do manuscrito. M. I. B. Senna participou da concepção e desenho do estudo, análise e interpretação dos dados e revisão crítica do manuscrito.

\section{Referências}

1. Almeida Filho N. Intersetorialidade, transdisciplinaridade e saúde coletiva: atualizando um debate em aberto. Rev Adm Pública 2000; 34:11-34.

2. Inojosa RM. Sinergia em políticas e serviços públicos: desenvolvimento social com intersetorialidade. Cad Fundap 2001; 22:102-10.

3. Junqueira LAP. A gestão intersetorial das políticas sociais e o terceiro setor. Saúde Soc 2004; 13:25-36.

4. Junqueira LAP. Articulações entre o serviço público e o cidadão. In: X Congreso Internacional del CLAD sobre da Reforma del Estado y de la Administración Pública. Santiago: Centro Latinoamericano de Administración para el Desarrollo; 2005. p. 18-21.

5. Mendes R, Akerman M. Intersetorialidade: reflexões e práticas. In: Fernandes JCA, Mendes $\mathrm{R}$, organizadores. Promoção da saúde e gestão local. São Paulo: Editora Hucitec/Cepedoc; 2007. p. 85-110.

6. Akerman M, Sá RF, Moysés S, Rezende R, Rocha D. Intersetorialidade? IntersetorialidadeS! Ciênc Saúde Coletiva 2014; 19:4291-300.

7. Ministério da Saúde. Portaria no 2.446, de 11 de novembro de 2014. Redefine a Política Nacional de Promoção da Saúde (PNPS). Diário Oficial da União 2014; 13 nov.

8. Andrade LOM. A saúde e o dilema da intersetorialidade. São Paulo: Editora Hucitec; 2006.

9. Ndumbe-Eyoh S, Moffatt H. Intersectoral action for health equity: a rapid systematic review. BMC Public Health 2013; 13:1056.

10. Garcia LMT, Maio IG, Santos TI, Folha CBJC, Watanabe HAW. Intersetorialidade na saúde no Brasil no início do século XXI: um retrato das experiências. Saúde Debate 2014; 38:966-80. 
11. Presidência da República. Decreto Presidencial no 6.286, de 5 de dezembro de 2007. Institui o Programa Saúde na Escola - PSE, e dá outras providências. Diário Oficial da União 2007; 6 dez.

12. Ministério da Saúde, Ministério da Educação. Manual instrutivo: Programa Saúde na Escola. Brasília: Ministério da Saúde/Ministério da Educação; 2013.

13. Ferreira IRC, Moysés SJ, França BHS, Carvalho ML, Moysés ST. Percepções de gestores locais sobre a intersetorialidade no Programa Saúde na Escola. Rev Bras Educ 2014; 19:61-76.

14. Jacoé NB, Aquino NM, Pereira SCL, Souza ES, Souza FC, Santana MS, et al. O olhar dos profissionais de uma Unidade Básica de Saúde sobre a implantação do Programa Saúde na Escola. Rev Méd Minas Gerais 2014; 24 Suppl 1:S43-8.

15. Graciano AMC. Percepção dos professores em relação à promoção da saúde em escolas do ensino fundamental [Tese de Doutorado]. Belo Horizonte: Universidade Federal de Minas Gerais; 2014.

16. Ferreira VSC, Silva LMV. Intersetorialidade em saúde: um estudo de caso. In: Hartz ZMA, Silva LMV, organizadores. Avaliação em saúde: dos modelos teóricos à prática da avaliação de programas e sistemas de saúde. Salvador: EDUFBA/Rio de Janeiro: Editora Fiocruz; 2014. p. 103-50.

17. Faria MSR. A intersetorialidade e os dilemas de sua prática no trabalho em saúde [Dissertação de Mestrado]. Belo Horizonte: Universidade Católica de Minas Gerais; 2013.

18. Silva KL, Sena RR, Akerman M, Belga SMM, Rodrigues AT. Intersetorialidade, determinantes socioambientais e promoção da saúde. Ciênc Saúde Coletiva 2014; 19:4361-70.

19. Moretti AC, Teixeira FF, Suss FMB, Lawder JAC, Lima LSM, Bueno RE, et al. Intersetorialidade nas ações de promoção de saúde realizadas pelas equipes de saúde bucal de Curitiba (PR). Ciênc Saúde Coletiva 2010; 15 Suppl 1:1827-34.

20. Ferreira IRC. Avaliação da intersetorialidade no Programa Saúde na Escola [Tese de Doutorado]. Curitiba: Pontifícia Universidade Católica do Paraná; 2012.

21. Prefeitura Municipal de Belo Horizonte. Portaria Conjunta SMED/SMSA/SMASAN no 003, de 12 de setembro de 2013. Institui o Grupo de Trabalho Intersetorial do Programa Saúde na Escola. Diário Oficial do Município 2013; 16 out.

22. Bardin L. L'analyse de contenu. Paris: PUF; 2013.

23. Ministério da Saúde. Portaria no 1.861, de 4 de setembro de 2008. Estabelece recursos financeiros pela adesão ao PSE para municípios com equipes de saúde da família, priorizados a partir do Índice de Desenvolvimento da Educação Básica - IDEB, que aderirem ao Programa Saúde na Escola - PSE. Diário Oficial da União 2008; 24 set.
24. Ministério da Saúde. Portaria no 3.146, de 17 de dezembro de 2009. Estabelece recursos financeiros para municípios com equipes de saúde da família, que aderirem ao Programa Saúde na Escola - PSE. Diário Oficial da União 2009; 18 dez.

25. Ministério da Saúde. Portaria Interministerial no 3.696, de 25 de novembro de 2010. Estabelece critérios para adesão ao Programa Saúde na Escola (PSE) para o ano de 2010 e divulga a lista de municípios aptos para manifestação de interesse. Diário Oficial da União 2010; 26 nov.

26. Ministério da Saúde. Portaria Interministerial no 1.910 , de 8 de agosto de 2011. Estabelece o Termo de Compromisso Municipal como instrumento para o recebimento de recursos financeiros do Programa Saúde na Escola (PSE). Diário Oficial da União 2011; 9 ago.

27. Ministério da Saúde. Portaria Interministerial no 1.413 , de 10 de julho de 2013. Redefine as regras e critérios para adesão ao Programa Saúde na Escola (PSE) por Estados, Distrito Federal e Municípios e dispõe sobre o respectivo incentivo financeiro para custeio de ações. Diário Oficial da União 2013; 11 jul.

28. Prefeitura Municipal de Belo Horizonte. Portaria Conjunta SMSA/SMED no 001, de 5 de novembro de 2008. Institui o Grupo de Trabalho Intersetorial para elaboração do Projeto do PSE. Diário Oficial do Município 2008; 7 nov.

29. Prefeitura Municipal de Belo Horizonte. Prefeitura promove formação para monitores do Programa Saúde na Escola. Diário Oficial do Município 2010; 20 jul.

30. Prefeitura Municipal de Belo Horizonte. Secretaria de Saúde qualifica atendimento do PSE nas escolas. Diário Oficial do Município 2011; 21 jul.

31. Prefeitura Municipal de Belo Horizonte. Programa Saúde na Escola atende todas as escolas da região oeste. Diário Oficial do Município 2011; 17 fev.

32. Cunill Grau N. La intersectorialidad en el gobierno y gestión de la política social. In: X Congreso Internacional del CLAD sobre da Reforma del Estado y de la Administración Pública. Santiago: Centro Latinoamericano de Administración para el Desarrollo; 2005. p. $1-52$.

33. Ferreira IRC, Vosgerau DSR, Moysés SJ, Moysés ST. Diplomas normativos do Programa Saúde na Escola: análise de conteúdo associada à ferramenta ATLAS TI. Ciênc Saúde Coletiva 2012; 17:3385-98.

34. Nascimento S. Reflexões sobre a intersetorialidade entre as políticas públicas. Serv Soc Soc 2010; 101:95-120.

35. Junqueira LAP, Inojosa RM, Komatsu S. Descentralização e intersetorialidade da gestão pública municipal no Brasil: a experiência de Fortaleza. In: XI Concurso de Ensayos del CLAD "El Tránsito de la Cultura Burocrática al Modelo de la Gerencia Pública: Perspectivas, Posibilidades y Limitaciones”. Caracas: Centro Latinoamericano de Administracion para el Desarrollo; 1997. p. 1-75. 
36. Sposati A. Gestão pública intersetorial: sim ou não? Comentários de experiência. Serv Soc Soc 2006; 85:133-41.

37. Pereira PAP. A intersetorialidade das políticas sociais na perspectiva dialética. In: Monnerat GL, Almeida NLT, Souza RG, organizadores. A intersetorialidade na agenda das políticas sociais. Campinas: Papel Social; 2014. p. 23-39.

38. Silva Junior AJ. Programa Saúde na Escola: limites e possibilidades intersetoriais [Tese de Doutorado]. Cuiabá: Universidade Federal de Mato Grosso; 2014.

39. Farias ICV. Análise da intersetorialidade no Programa Saúde na Escola no município de Olinda - PE [Dissertação de Mestrado]. Recife: Universidade Federal de Pernambuco; 2014.

40. Sousa MC, Esperidião MA, Medina MG. A intersetorialidade no Programa Saúde na Escola: avaliação do processo político-gerencial e das práticas de trabalho. Ciênc Saúde Coletiva 2017; 22:1781-90.
41. Monnerat GL, Souza RG. Intersetorialidade e políticas públicas: um diálogo com a literatura atual. In: Monnerat GL, Almeida NLT, Souza $\mathrm{RG}$, organizadores. A intersetorialidade na agenda das políticas sociais. Campinas: Papel Social; 2014. p. 41-54.

42. Brasil. Lei no 9.394, de 20 de dezembro de 1996. Estabelece as diretrizes e bases da educação nacional. Diário Oficial da União 1996; 23 dez.

43. Mannheimer LN, Gulis G, Lehto J, Östlin P. Introducing health impact assessment: an analysis of political and administrative intersectoral working methods. Eur J Public Health 2007; 7:526-31. 


\section{Abstract}

This study evaluated the implementation of the School Health Program (PSE in Portuguese) in the city of Belo Horizonte, Minas Gerais State, Brazil, the mechanisms favoring inter-sector action, and municipal administrators' perceptions concerning inter-sector collaboration. A case study was developed with document search and the application of an online questionnaire. The document search analyzed federal and municipal legislation on the PSE published in the Federal Register and Municipal Register and news on the program published in the Municipal Register. A semistructured online questionnaire was completed by 30 municipal administrators. Content analysis was used on the qualitative data from the document search and questionnaire. The quantitative data were interpreted by descriptive analysis using Stata v. 13. Integrative mechanisms were identified in the federal and municipal provisions and in the unique PSE model adopted by the city. These mechanisms can potentially promote permanent linkage between health and education. However, the study with municipal administrators showed limited use of these mechanisms and the predominance of a sector-based logic in the program. There was evident difficulty in developing intersector collaboration in the program's activities. The potentialities of inter-sector action identified in the official documents and described in the institutional news failed to reverberate in either the administrators' practices or the impact on the schools' territories.

Intersectorial Collaboration; School Health; Health Promotion; Public Policies

\section{Resumen}

Este estudio evaluó la implementación y ejecución del Programa Salud en la Escuela (PSE) en el municipio de Belo Horizonte, Minas Gerais, Brasil, así como los mecanismos que favorecen la acción intersectorial y la percepción de los gestores municipales sobre la intersectorialidad. Se desarrolló un estudio de caso mediante una investigación documental y la aplicación de un cuestionario electrónico. La investigación documental analizó los decretos federales y municipales sobre el PSE, publicados en el Boletín Oficial de la Unión y en el Boletín Oficial del Municipio, así como noticias sobre el programa publicados en el Boletín Oficial del Municipio. Un cuestionario semiestructurado online se aplicó a 30 gestores municipales. El análisis de contenido se usó para los datos cualitativos de la investigación documental y del cuestionario. Los datos cuantitativos se interpretaron mediante un análisis descriptivo, realizado por el programa Stata v. 13. Se identificaron mecanismos integradores en las normativas federales y municipales, así como en el modelo singular de PSE adoptado por el municipio. Estos mecanismos son potencialmente promotores de la coordinación permanente entre salud y educación. La investigación con los gestores demostró, no obstante, una escasa utilización de esos mecanismos y el predominio de la lógica sectorial en esa instancia del programa. Se percibieron dificultades en la construcción de la intersectorialidad, así como el desarrollo de las acciones del PSE. Las potencialidades de la acción intersectorial identificadas en los textos oficiales, $y$ descritas en los boletines institucionales, no repercutieron en las prácticas de los gestores ni en la actuación sobre los territorios.

Colaboración Intersectorial; Salud Escolar;

Promoción de la Salud; Políticas Públicas
Recebido em 18/Jun/2017

Versão final reapresentada em 10/Out/2017

Aprovado em 04/Dez/2017 